\title{
Density functional embedding for periodic and nonperiodic diffusion Monte Carlo calculations
}

\author{
Katharina Doblhoff-Dier* and Geert-Jan Kroes \\ Leiden Institute of Chemistry, Gorlaeus Laboratories, Leiden University, Post Office Box 9502, 2300 RA Leiden, Netherlands \\ Florian Libisch \\ Institut for theoretische Physik, Technische Universität Wien, Austria
}

(Received 25 April 2018; revised manuscript received 3 July 2018; published 23 August 2018)

\begin{abstract}
We present density functional embedding for diffusion Monte Carlo calculations (DMC). Using two test systems, an $\mathrm{H}$ chain and a Be slab, we demonstrate the feasibility and show that the approach can give high-quality results. DMC embedded in potentials from density functional embedding theory thus presents an alternative to conventional embedded quantum chemical methods, such as embedded complete active space calculations. This is especially true for cases in which the cluster size becomes too large for accurate wave-function theory based methods or for cases in which basis-set superposition errors become dominant. Furthermore, we show that embedded DMC can easily be used for systems in which the cluster of interest is a one-dimensional or two-dimensional periodic object and cannot be described as isolated impurity. This extends the applicability of the embedding approach to new fields.
\end{abstract}

DOI: 10.1103/PhysRevB.98.085138

\section{INTRODUCTION}

In the effort to accurately assess the electronic properties of atomic and molecular systems of ever increasing sizes, two obvious strategies exist: one can either try to improve the numerical scaling of high-level methods or increase the accuracy of low-level methods. Examples of the former include massively parallel implementations of coupled cluster approaches [1], linear scaling coupled cluster [2], and quantum Monte Carlo (QMC) methods [3-5]. The latter strategy strongly targets the improvement of density functional theory approaches and includes the development of more accurate exchange-correlation functionals, including improved semilocal functional [6-11], van der Waals functionals [12-15], improved hybrid functionals $[16,17]$, and the use of machinelearning algorithms $[13,18]$.

A third, promising, alternative is to combine the advantages of several methods in one calculation, by partitioning the system of interest into a (small) site of interest and an environment. The site of interest is then treated with a high-level approach and is embedded in an environment that can be considered sufficiently well described at a lower level of theory. Apart from the choice of the two methods, crucial questions are how to choose the partitioning and how to manage the interaction between cluster and environment. Different types of systems typically require a different treatment of the environment: for ionic crystals, an embedding in point charges is typically sufficient $[19,20]$; for biochemical systems, combined quantum mechanics/molecular mechanics (QM/MM) [21] will often be the method of choice, while solid-state systems may be better described using density functional embedding [22-26]. More involved schemes such as density-matrix embedding

*Corresponding author: k.doblhoff-dier@umail.leidenuniv.nl
[27-29] or projection-based methods [30-33] allow for a more exact treatment of the cluster-environment interaction, in particular the kinetic energy. However, such schemes require additional operators in the Hamiltonian of the embedded cluster, instead of merely a local potential, possibly making their implementation for different electronic structure methods more challenging.

In spite of the improvements offered by modern embedding methods, the size of the cluster described at a higher level of theory remains a crucial "parameter" that needs to be converged. Often, the cluster sizes required become too large to allow for a highly accurate description with conventional quantum chemistry methods. The exploration of new methods to be used inside an embedding calculation therefore becomes of interest. In this paper, we will discuss and investigate the combination of diffusion Monte Carlo (DMC), a quantum Monte Carlo method, with embedding theories.

In the past, diffusion Monte Carlo has been coupled to the reaction field of classical polarizable dipoles in a QMC/MMpol [34] approach, to continuum solvation from joint density functional theory [35] and, for molecular systems, to wave function in DFT embedding schemes [26]. Here, we will demonstrate the successful use of embedding potentials from density functional embedding theory (DFET) [36] in DMC calculations, also including periodic DMC calculations embedded in DFT.

Density functional embedding has previously been used in conjunction with clusters described by second-order multireference many-body theory [37], complete active space (CAS), CASPT2, and configuration interaction (CI) calculations [38]. Briefly, DFET employs a single, local embedding potential $V_{\mathrm{emb}}$ to mediate the interaction between cluster and environment. Other than conventional DFT embedding schemes, DFET does not rely on approximate kinetic energy functionals to determine $V_{\mathrm{emb}}$. After determining $V_{\mathrm{emb}}$ on the level of 
density functional theory, the embedded cluster can be solved using high-level methods such as DMC. The use of DMC in conjunction with DFET would allow for the treatment of larger clusters due to the better scalability of DMC compared to the previously employed quantum chemical methods.

Embedding DMC calculations is of particular interest for the solid state, including metals and their interfaces, since both DMC and DFET are well suited for treating periodic boundary conditions. One may thus treat systems in which the cluster of interest is one-dimensional (1D) and two-dimensional (2D) periodic instead of an isolated impurity, yielding an important extension to conventional density functional embedding. As an example thereof, we consider a Be slab in this paper.

Furthermore, DMC could overcome problems faced by conventional quantum chemistry methods with respect to finite basis-set errors, which can be particularly large in metallic systems. Since DMC is quasi-basis-set free, it will typically not suffer from excessive basis-set related errors (compare Refs. [39,40]).

On a more general level, the integration of DFET embedding potentials into DMC may thus provide the possibility to access properties of systems that are otherwise hard to determine at sufficiently high accuracy, such as reaction barriers for dissociative chemisorption [41-43] and molecular chemisorption energies $[15,44]$ on metals.

There is, however, one concern when using DMC to describe a cluster embedded in a density functional embedding potential: the problematic convergence of the optimized effective potential approaches [45] involved in determining the DFET embedding potential results in numerical noise that manifests as small-scale oscillations. These nonphysical oscillations in the embedding potential will likely not influence the cluster calculation if the cluster is described with methods that use a finite basis set (e.g., a Gaussian basis) since the basis set will generally not be flexible enough to resolve them. By contrast, DMC has no basis-set related restrictions, other than those entering the fixed-node approximation and the locality approximation. Hence, a DMC wave function can follow all oscillations no matter how small. In this paper, we thus not only present the implementation of potential functional embedding for the QMC code CASINO, but also test the actual applicability of the approach for two simple test systems, a hydrogen chain and a Be slab, thus validating the approach.

The paper is structured as follows: First, we give a short overview of the methods used, including diffusion Monte Carlo (Sec. II A) and density functional embedding theory (Sec. II B). Then, we present our results, first for the $\mathrm{H}$ chain (Sec. III) and then for the cohesive energy of Be as computed from 2D periodic slab calculations (Sec. IV). The paper concludes with a summary and outlook.

\section{METHODS}

\section{A. Diffusion Monte Carlo}

Diffusion Monte Carlo belongs to a class of methods that aim at solving the electronic Schrödinger equation for the ground state using stochastic methods. In particular, DMC makes use of the imaginary-time Schrödinger equation.
Since excellent reviews on the DMC method have been published elsewhere $[3,4,46,47]$, we will only give a very brief description here.

In DMC, the imaginary-time Schrödinger equation is recast into a drift diffusion and branching equation of a set of electronic configurations ("walkers"). At every point in imaginary time, the walkers stochastically represent the distribution

$$
f(\{\mathbf{r}\}, t)=\Psi_{T}(\{\mathbf{r}\}) \cdot \Psi(\{\mathbf{r}\}, t),
$$

where $\Psi_{T}(\{\mathbf{r}\})$ is an initial trial wave function depending on the electronic coordinates $\{\mathbf{r}\}$ and $\Psi(\{\mathbf{r}\}, t)$ is the imaginary-time propagation of $\Psi_{T}(\{\mathbf{r}\})$ subject to the fixed-node constraint, i.e., the nodes of the wave function remain fixed in the $3 \mathrm{~N}$ dimensional configuration space (with $N$ being the number of electrons in the system). After the equilibration of $\Psi(\{\mathbf{r}\}, t)$, i.e., for $t>t_{\text {equil }}$, such that

$$
\Psi(\{\mathbf{r}\}, t) \approx \Psi(\{\mathbf{r}\}, t \rightarrow \infty) \equiv \Psi_{0}^{\mathrm{fn}}(\{\mathbf{r}\}) \quad \forall t>t_{\text {equil }},
$$

the fixed-node ground-state energy corresponding to the ground state under the fixed-node constraint $\Psi_{0}^{\mathrm{fn}}(\{\mathbf{r}\})$ is given by

$$
E_{0}^{\mathrm{fn}}=\left\langle E_{L}(\{\mathbf{r}\})\right\rangle,
$$

with $E_{L}(\{\mathbf{r}\})=\frac{\hat{H} \Psi(\{\mathbf{r}\})}{\Psi(\{\mathbf{r}\})}$, where $\hat{H}$ is the electronic Hamiltonian.

The propagation of the walkers is performed in finite time steps $\tau$, the converged length of which depends on the system and the quality of the trial wave function $\Psi_{T}(\{\mathbf{r}\})$. The trial wave function $\Psi_{T}(\{\mathbf{r}\})$ is typically given by

$$
\Psi_{T}(\{\mathbf{r}\})=S(\{\mathbf{r}\}) \cdot e^{J(\{\mathbf{r}\})},
$$

where $S(\{\mathbf{r}\})$ is a wave function expressed in one or more [40,48-50] Slater determinants (for example, from a HartreeFock calculation, a DFT calculation or a CI calculation) and $e^{J(\{\mathbf{r}\})}$ is the Jastrow factor. The Jastrow function $J$ is a function of electron-electron distances $r_{i j}$ and ion-electron distances $\left(r_{I i}\right.$ and $\left.r_{I j}\right)$ and serves to introduce many particle correlation effects. This function is typically parametrized by tens to a few hundreds of parameters, which are optimized in a variational Monte Carlo calculation prior to a DMC calculation by either minimizing the variance of the local energies $E_{L}[51,52]$ or by minimizing the variational energy $\left\langle E_{L}\right\rangle$ itself $[53,54]$.

For our calculations, we use a Jastrow function given by a sum of electron-electron terms $u$, electron-nucleus terms $\chi$, and, for the $\mathrm{H}_{4}$ chain, electron-electron-nucleus three-body terms $f$ :

$$
\begin{aligned}
J\left(\left\{\mathbf{r}_{i}\right\},\left\{\mathbf{r}_{I}\right\}\right)= & \sum_{I, i} \chi\left(r_{i I}\right)+\sum_{i, j \neq i} u\left(r_{i j}\right) \\
& +\sum_{I, i, j \neq i} f_{I}\left(r_{i I}, r_{j I}, r_{i j}\right),
\end{aligned}
$$

where $i$ and $j$ enumerate electrons, $I$ enumerates ions, and $r_{x y}$ is the distance in real space between particle $x$ and particle $y$.

The functions $\chi\left(r_{i I}\right)$ and $u\left(r_{i j}\right)$ are parametrized by polynomials of degree $N$ multiplied by a smooth cutoff function

$$
P_{N}(x)\left(x-L_{x}\right)^{3} \Theta\left(L_{x}-x\right),
$$


where $P_{N}(x)$ is a polynomial of degree $N, L_{x}$ denotes the cutoff length, and $\Theta$ is the Heaviside theta function [46]. In case of the $u$ term, the polynomial coefficients are restricted to satisfy the electron-electron cusp condition. The three-body function $f\left(r_{i j}, r_{i I}, r_{j I}\right)$ is given equivalently as a polynomial in the three independent variables multiplied with a cutoff function in $r_{i I}$ and $r_{j I}$.

For the present calculations, we use $N=5$ for the $u$ and $\chi$ terms and $N=2$ in the $f$ term, if used. Different parameters are used for spin-up and -down electrons in the $\chi$ term and for the different spin pairs in the $u$ term. The optimizable parameters (i.e., the cutoff lengths $L_{x}$ and the polynomial coefficients) are optimized by minimizing the variance of the local energies.

For all quantum Monte Carlo calculations, we use the software package CASINO [46]. The extension to allow for embedding potentials has been added to the code by us and will shortly become available in the beta version of CASINO. To allow for an interpolation of the potential, it is reexpressed in cubic splines.

\section{B. Density functional embedding theory}

Density functional embedding theory (DFET) [24] belongs to the group of orbital free embedding schemes, in which only the density is partitioned into two or more systems. In contrast to previously developed orbital free embedding schemes, such as the frozen density embedding by Wesolowski and Warshel [22], DFET does not employ any kinetic energy density functionals. Furthermore, for every choice of electronic and nuclear partitioning, DFET guarantees a unique partitioning of the densities by requiring that the two subsystems share the same embedding potential $V_{\mathrm{emb}}(\mathbf{r})$ with

$$
\rho_{1}\left[V_{\mathrm{emb}}\right]+\rho_{2}\left[V_{\mathrm{emb}}\right]=\rho_{\text {full }},
$$

where $\rho_{1}$ and $\rho_{2}$ are the densities of the first and the second subsystems (i.e., the cluster and the environment) obtained under the influence of the embedding potential $V_{\text {emb. The }}$ density $\rho_{\text {full }}$ is the density of the total system. (Here and in most of the following, we omit the dependence of $\rho$ and $V_{\mathrm{emb}}$ on $\mathbf{r}$ to improve readability.)

The embedding potential is optimized self-consistently on the density functional theory level by maximizing the extended Wu-Yang functional [55] as function of $V_{\mathrm{emb}}$ :

$$
\begin{aligned}
W\left[V_{\mathrm{emb}}\right]= & E_{1}\left[\rho_{1}\right]+E_{2}\left[\rho_{2}\right] \\
& +\int V_{\mathrm{emb}}\left(\rho_{1}+\rho_{2}-\rho_{\mathrm{full}}\right) d \mathbf{r}+w\left[V_{\mathrm{emb}}\right] .
\end{aligned}
$$

(Note that $\rho_{1}+\rho_{2}-\rho_{\text {full }}$ is not zero as long as the embedding potential is not converged and that $W$ and $w$ are not explicitly $\mathbf{r}$ dependent.) The penalty term $w\left[V_{\mathrm{emb}}\right]$ is introduced into the $\mathrm{Wu}$-Yang functional to regularize the potential by penalizing wild oscillations in the embedding potential

$$
w\left[V_{\mathrm{emb}}\right]=-\lambda \int V_{\mathrm{emb}} \nabla^{2} V_{\mathrm{emb}} d \mathbf{r},
$$

where $\lambda$ is a constant that scales the weight of the penalty function.

The resulting embedding potential can then be used to determine the ground-state energy $E_{\text {cluster }}^{X}\left[V_{\text {emb }}\right]$ of subsystem
1 (the cluster) in the presence of $V_{\mathrm{emb}}$, at a different level of theory $X$. The total energy $E_{\text {tot }}^{\mathrm{DFET}}$ is then given by

$$
E_{\text {tot }}^{\mathrm{DFET}}=E_{\text {full }}^{\mathrm{DFT}}-E_{\text {cluster }}^{\mathrm{DFT}}\left[V_{\mathrm{emb}}\right]+E_{\text {cluster }}^{X}\left[V_{\mathrm{emb}}\right],
$$

where $E_{i}^{M}$ denotes the energy obtained for the system $i$, $i \in\{$ full, cluster $\}$ at the level of theory $M$, and the functional dependence $E\left[V_{\mathrm{emb}}\right]$ indicates the fact that the ground-state energy is calculated in the presence of the embedding potential. This definition of $E_{\mathrm{tot}}^{\mathrm{DFET}}$ automatically includes a first-order correction to the interaction energy, thus accounting for the change of the cluster density from $\rho_{\text {cluster }}^{\mathrm{DFT}}$ to $\rho_{\text {cluster }}^{\mathrm{X}}$, as shown in Ref. [24].

Overall, DFET is very similar to the DFT in DFT (DFT/DFT) freeze and thaw algorithm proposed for frozen density embedding (FDE) [56], albeit without the need of an approximate kinetic energy functional or the need to deduce the orbitals from an optimized effective potential [23]. Using the algorithm presented above, a relatively stable optimization of the embedding potential can be achieved. Furthermore, the only change in evaluating the ground-state energy of the cluster is the additional scalar embedding potential $V_{\mathrm{emb}}$, making the connection with different high-level approaches comparatively easy. It should be kept in mind, however, that DFET calculations are not self-consistent in the sense that the embedding potential $V_{\text {emb }}$ does not react to the improved cluster density $\rho_{\text {cluster }}^{X}$ [except for a first-order correction, see Eq. (10)]. Instead, the embedding potential is only determined once, at a pure DFT level. This is in contrast to iterative wave-function/DFT schemes, which have been proposed as an extension to freeze and thaw FDE algorithms [57]. In these wave-function/DFT schemes, the embedding potential is constructed self-consistently for a cluster described by a wave-function method and an environment described by DFT. Extending these wave-function/DFT schemes to DMC/DFT might, however, be nontrivial since the system density, which enters the iteratively improved embedding potential in these schemes, is only sampled stochastically in DMC. Although smooth representations of the density can be obtained by projecting onto basis functions [58] or by sampling in discrete Fourier space, it remains questionable and a matter of future investigation whether these representations can be made accurate enough. Previous "DMC"/DFT applications were therefore based on embedding potentials that were self-consistently created at a mixed CASPT2/DFT level [26] making the extension to periodic systems challenging. Similar concerns also hold true for potential functional embedding theory [25], which would also allow for a self-consistent embedding at a wave-function/DFT level. At present, and in spite of its restrictions, we therefore view DFET to be the most suitable embedding scheme if a connection to DMC is sought for solid-state systems.

All embedding potentials used in this work have been created with the embedding code for ABINIT available from the group of E. Carter with some minor modifications.

\section{III. $\mathrm{H}_{4}$ CHAIN}

As first test system, we will consider a chain of four $\mathrm{H}$ atoms in a box with the $\mathrm{H}$ atoms represented by Trail-Needs pseudopotentials. The property of interest is the change in 
(a)

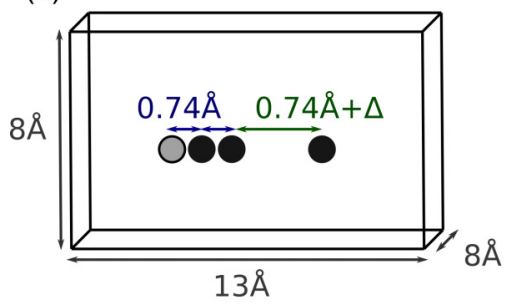

(b)

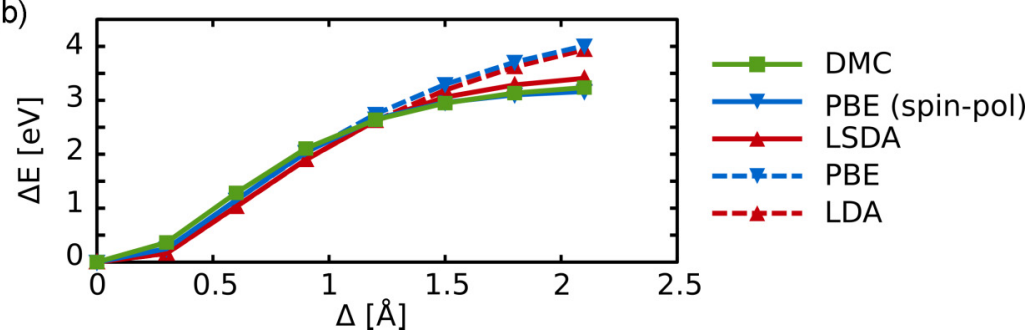

FIG. 1. (a) Sketch of the $\mathrm{H}_{4}$ chain. The light gray atom is replaced by an embedding potential in the embedded cluster calculations. (b) Change in binding energy for different values of $\Delta$, where $\Delta$ is defined in subplot (b). The error bars of the DMC results are not shown, but they are all smaller than $10 \mathrm{meV}$ and are thus negligible.

energy when one of the outermost bonds is stretched by an amount $\Delta$ as shown in Fig. 1(a). Before considering results in which the atom farthest from the bond stretch is replaced by an embedding potential, we will briefly discuss the results obtained when treating the full system at one level of theory.

Figure 1(b) shows the change in energy for (spin-polarized and non-spin-polarized) DFT calculations using different functionals when stretching the outermost bond by an amount $\Delta$. For the DFT calculations, spin polarization clearly becomes important for bond stretches larger than $\Delta=1.2 \AA$ [see Fig. 1(b)]. Figure 1(b) also shows the corresponding DMC energies. The DMC caclulations were thereby performed using a time step of $\tau=0.02 \mathrm{au}$. The Slater part of the trial wave function $S(\{\mathbf{r}\})$ is given by a single Slater determinant taken from spin-unpolarized calculations with the LDA functional [59] and the three-dimensional (3D) periodicity of the box is kept for consistency with the DFT results. Due to the relatively large box size, this can be expected to have little effect. The DMC calculations agree very well with results of spin-polarized calculations using the PBE functional [60], despite the fact that they are based on a spin-unpolarized $S(\{\mathbf{r}\})$. Apparently, the DMC calculations can recover the effects of spin polarization to a certain extent.

To test the embedding implementation for $\mathrm{DMC}$, the $\mathrm{H}$ atom farthest from the stretched bond was replaced by an embedding potential. Both fragments were thereby kept neutral and the embedding potential was based on spin-unpolarized LDA calculations and a smoothing parameter of $\lambda=5 \times 10^{-5}$. The calculations were considered converged when the maximum density difference was less than $1 \times 10^{-3}$ a.u.

Since there are concerns about the possible harmfulness of the "wiggles" in the embedding potential to accurate DMC calculations, we first investigate this possibility in more detail: The concerns just mentioned are rooted in the fact that DMC is quasi-basis-set free [apart from influences of the basis set used to construct $S(\{\mathbf{r}\})$ on the fixed-node approximation] and can thus easily respond to short-range oscillations in the embedding potential. We can simulate this effect in a pure DFT approach, thus allowing for a cheap proof of principle. To this end, we proceed a follows: first, we construct embedding potentials for several values of $0 \leqslant \Delta \leqslant 1.2 \AA$ at a plane-wave cutoff of $E_{\text {cut }}=2000 \mathrm{eV}$. At this $E_{\text {cut }}$, the DFT calculations are highly converged (total energies converged to about $20 \mathrm{meV}$ ). We then use these embedding potentials in DFT calculations with a higher plane-wave cutoff of $E_{\text {cut }}=3100 \mathrm{eV}$, thus simulating the effect of having a full basis. The integrated density difference $\int \Delta \rho d \mathbf{r}$ of

$$
\Delta \rho=\rho_{\text {cluster }}\left(E_{\text {cut }}^{i}\right)+\rho_{\text {env }}\left(E_{\text {cut }}^{i}\right)-\rho_{\text {full }}\left(E_{\text {cut }}^{i}\right),
$$

the maximum density difference, the total energy of the cluster $E_{\text {cluster }}^{\mathrm{DFT}}\left[V_{\mathrm{emb}}\right]$ [i.e., the energy of the cluster plus its interaction with the embedding potential as defined in Eq. (10)] and the energy of the cluster $\tilde{E}_{\text {cluster }}^{\mathrm{DFT}}\left[V_{\text {emb }}\right]$ :

$$
\tilde{E}_{\text {cluster }}^{\mathrm{DFT}}\left[V_{\mathrm{emb}}\right]=E_{\text {cluster }}^{\mathrm{DFT}}\left[V_{\mathrm{emb}}\right]-\int V_{\mathrm{emb}}(r) \rho_{\text {cluster }}(r) d \mathbf{r}
$$

were then examined. Hereby, $\rho_{x}\left(E_{\text {cut }}^{i}\right)$ denotes the density of the embedded cluster, the embedded environment, and the full system computed at a given plane-wave cutoff energy $E_{\text {cut }}^{i}$, for $x \in\{$ cluster, env, full $\}$, respectively.

Using this approach, the maximum density difference increases from below $1 \times 10^{-3}$ au (the value to which it was converged at $E_{\text {cut }}^{1}=2000 \mathrm{eV}$ ) to about $2 \times 10^{-3}$ au at $E_{\text {cut }}^{2}=3100 \mathrm{eV}$ for all values of $\Delta$ (see Fig. 2 and Table I for the case $\Delta=0 \AA$ ). The integrated density difference also increases with increasing $E_{\text {cut }}$, but not as much as the maximum density difference (see Table I for the case $\Delta=$ $0 \AA$ A). The largest density differences arise close to the nucleus replaced by the embedding potential (see Fig. 2). Such a

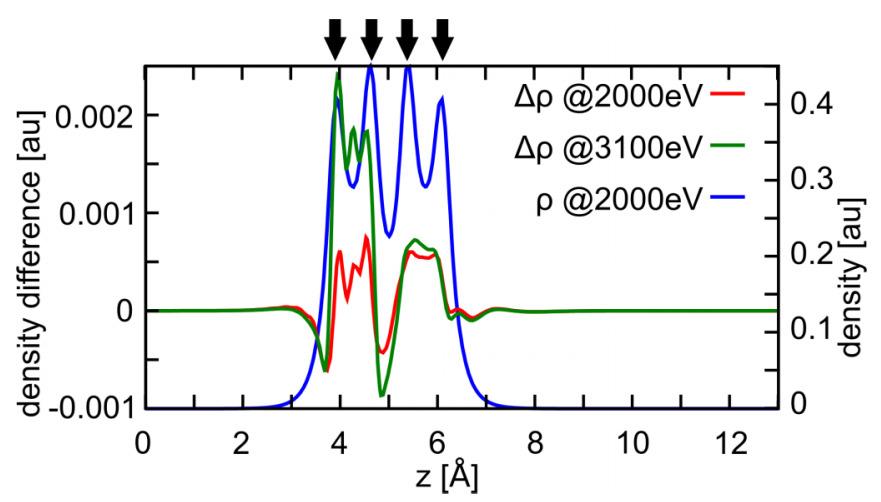

FIG. 2. Cut of the density differences $\Delta \rho$ between the sum of the cluster density and the environment density, and the density of the full calculation along the chain of $\mathrm{H}$ atoms for different values of $E_{\mathrm{cut}}$ (left $y$ axis). The total density is shown for reference (right $y$ axis). The positions of the $\mathrm{H}$ atoms in the box are indicated by black arrows. The leftmost atom is the one that is replaced by an embedding potential. 
TABLE I. Density differences as computed from Eq. (11) for the $\mathrm{H}_{4}$ chain separated into cluster and environment at $\Delta=0 \AA$.

\begin{tabular}{lcc}
\hline \hline & $E_{\text {cut }}=2000 \mathrm{eV}$ & $E_{\text {cut }}=3100 \mathrm{eV}$ \\
\hline $\max (\Delta \rho)$ & $0.8 \times 10^{-3} \mathrm{au}$ & $2.4 \times 10^{-3} \mathrm{au}$ \\
$\int \Delta \rho d \mathbf{r}$ & $2.3 \times 10^{-2} \mathrm{au}$ & $2.4 \times 10^{-2} \mathrm{au}$ \\
\hline \hline
\end{tabular}

behavior can be expected: since the embedding potential was constructed at $E_{\text {cut }}^{1}$, the refined ground-state density for the cluster+environment calculations at $E_{\text {cut }}^{2}>E_{\text {cut }}^{1}$ will react to the low spatial resolution of $V_{\text {emb }}$. Short-length oscillations observed in the density of the full system will not be captured in the cluster+environment calculations resulting in the observed increase in maximum density difference. While this behavior likely introduces an additional basis-set effect in the DMC calculations, we do not observe qualitative changes in the groundstate density at higher $E_{\text {cut }}$ that are caused by oscillations in the embedding potential. This is likely due to the suppression of sharp oscillations in the density by corresponding high kinetic energy contributions. We conclude that the quasi-basis-set free nature of our QMC calculations poses no fundamental obstacle towards embedded QMC-in-DFT approaches.

As a second test, we also consider the basis-set effects on the total energy of the cluster $E_{\text {cluster }}^{\mathrm{DFT}}\left[V_{\mathrm{emb}}\right]$ as defined in Eq. (10). This value is of particular interest since it directly enters the evaluation of the total energy $E_{\mathrm{tot}}^{\mathrm{DFET}}$ of the embedded system. For $E_{\text {cluster }}^{\mathrm{DFT}}\left[V_{\mathrm{emb}}\right]$, we observe very small changes of less than $5 \mathrm{meV}$ when increasing $E_{\text {cut }}$ from 2000 to $3100 \mathrm{eV}$. This is curious since these changes are significantly smaller than the changes of $\sim 20 \mathrm{meV}$ that were observed for the total energy of the full system. To shed more light on this, we also consider the energy of the cluster only $\tilde{E}_{\text {cluster }}^{\mathrm{DFT}}\left[V_{\mathrm{emb}}\right]$ :

$$
\tilde{E}_{\text {cluster }}^{\mathrm{DFT}}\left[V_{\mathrm{emb}}\right]=E_{\text {cluster }}^{\mathrm{DFT}}\left[V_{\mathrm{emb}}\right]-\int V_{\mathrm{emb}}(r) \rho_{\text {cluster }}(r) d \mathbf{r} .
$$

The changes in $\tilde{E}_{\text {cluster }}^{\mathrm{DFT}}\left[V_{\mathrm{emb}}\right]$ are in the range of 20 to $30 \mathrm{meV}$, in good agreement with the energy changes observed for the total system. Apparently, there is a certain amount of error cancellation in the change of the total cluster energy $E_{\text {cluster }}^{\mathrm{DFT}}\left[V_{\mathrm{emb}}\right]$ when going from small $E_{\text {cut }}$ to large $E_{\text {cut }}$. Overall, the small changes observed for an increased basis set size are reassuring. We thus proceed to actually use the embedding potential obtained at $E_{\text {cut }}=2000 \mathrm{eV}$ in embedded DMC calculations.

Figure 3(a) shows a comparison of the DMC energy obtained for the full system with the DMC energy obtained in an embedded cluster calculation. Since we do not consider spin effects in the embedding, we do not expect the embedding framework to be able to capture the spin effects that become important at large values of $\Delta$ [see Fig. 1(a)]. We therefore focus on the region with small $\Delta \leqslant 0.9 \AA$.

The energy difference of the embedded calculation to the benchmark full DMC calculations shows remarkable agreement up to the point were spin contributions become essential (at a spacing $\Delta>0.9 \AA$ ) [see Fig. 3(b)]. In this range of bond lengths, the embedded DMC calculations also perform considerably better than LDA or PBE calculations for the full system [see Fig. 3(b)]. To prove that this agreement is, indeed, due to the embedding formalism, we also consider a naive "embedding" scheme that neglects any interaction of the cluster to its environment

$$
E_{\text {tot }}^{\mathrm{corr}}=E_{\text {full }}^{\mathrm{DFT}}-E_{\text {cluster }}^{\mathrm{DFT}}\left[V_{\mathrm{emb}}=0\right]+E_{\text {cluster }}^{\mathrm{DMC}}\left[V_{\mathrm{emb}}=0\right] .
$$

The DMC calculation $E_{\text {cluster }}^{\mathrm{DMC}}\left[V_{\mathrm{emb}}=0\right]$ is thereby based on Slater wave functions from DFT calculations of the cluster without embedding. If this scheme were to give similar results to DMC embedded in a DFET(LDA) potential, this would clearly indicate that no elaborate DFET embedding is needed for this system, making statements about the role of the wiggles in $V_{\text {emb }}$ useless. Any agreement of embedded DMC with the full DMC results could then not be viewed as a sign that DFET of DMC can provide high-quality results. In the present case, the nonembedded DMC calculations perform significantly worse than DFET, clearly indicating the need of an embedding potential.

The attentive observer may have noticed that it is only the embedded DMC calculation that seems to suffer from spinpolarization effects, while the full DMC calculations closely follow spin-polarized PBE for this system. We explain this by the reduced flexibility of the wave function in the embedded DMC calculations as compared to the full calculation: by
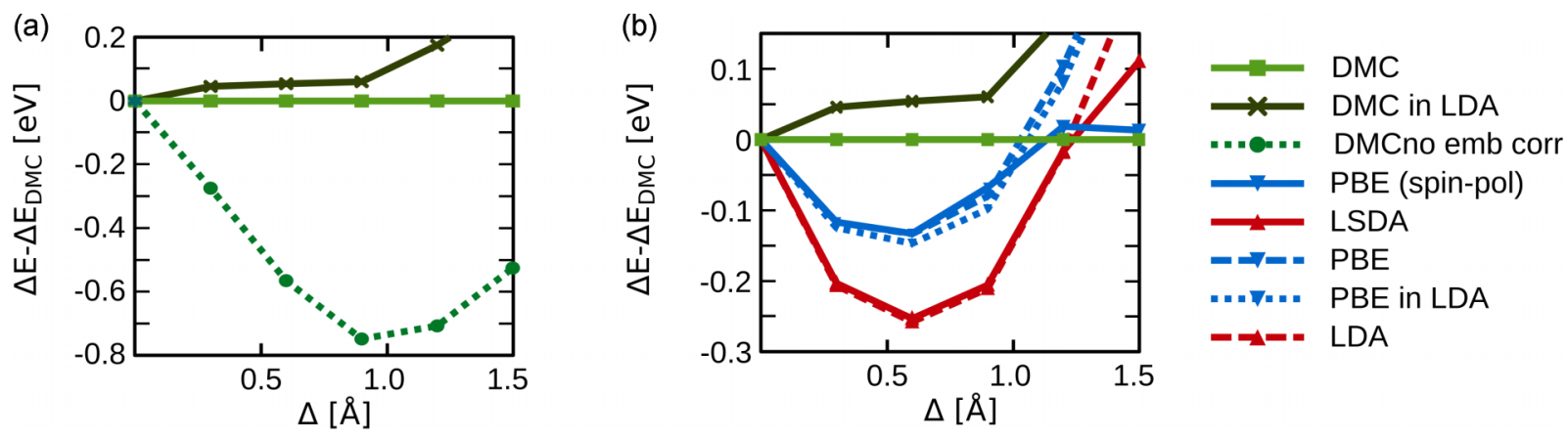

FIG. 3. Energy change associated with the bond stretch in the $\mathrm{H}_{4}$ chain: (a) DMC results for the full system compared to embedded DMC (DMC in LDA) results and DMC results for the cluster without embedding potential (see text for details). (b) Comparison of embedded DMC results with embedded DFT results. The error bars of the DMC results are not shown, but they are all smaller than 10 meV and are thus negligible. 


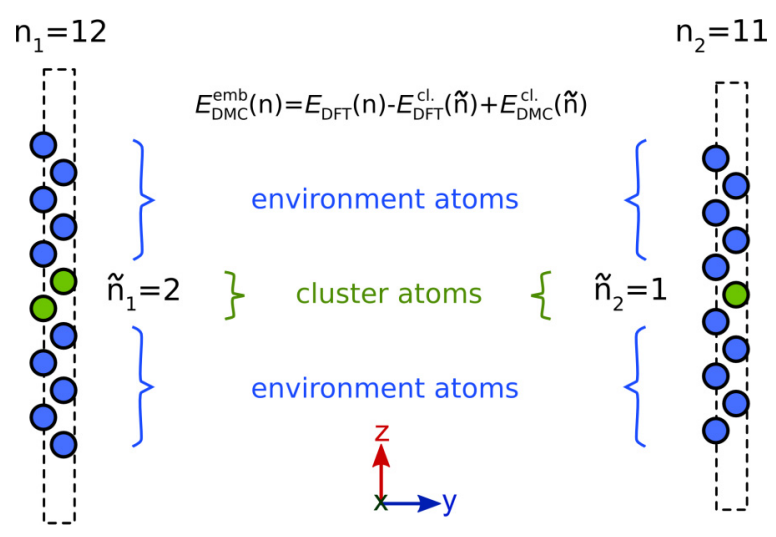

FIG. 4. Sketch of the geometries used to determine the cohesive energy [see Eq. (15)] of Be. The slabs are periodic in the $x-y$ plane.

replacing one of the electrons with a DFT-based embedding potential, the flexibility of this electron is lost in DMC, which can no longer relax towards the true (fixed-node) solution.

Overall, this example demonstrates the applicability of DFET to DMC. The deviation of the embedded DMC calculation from the full DMC calculations for $\Delta>0.9 \AA$ is most likely due to spin effects, as corroborated by the comparison between LDA and LSDA (as well as PBE and spin-polarized PBE) and the embedded DMC results in Fig. 3(b). Within the range of applicability (which is limited by the embedding potential being computed for a non-spin-polarized system), embedded DMC thus seems to be capable of giving good results (on an accuracy scale of better than $65 \mathrm{meV}$ for $\Delta \leqslant$ $0.9 \AA)$.

\section{COHESIVE ENERGY OF BERYLLIUM}

As mentioned in the Introduction, an advantage of embedded DMC calculations over other embedded correlated wave-function approaches (embedded complete active space or embedded configuration interaction) is that in addition to the fact that the cluster may be chosen larger, DMC is naturally capable of performing periodic calculations. It thus becomes possible to embed $1 \mathrm{D}$ or $2 \mathrm{D}$ periodic structures in a DFT environment. As a proof of principle, we compute the cohesive energy of bulk beryllium from

$$
E_{\mathrm{coh}}=\frac{E\left(n_{1}\right)-E\left(n_{2}\right)}{\left(n_{1}-n_{2}\right) N_{\mathrm{at}}}-E_{\mathrm{atom}},
$$

where $E(n)$ is the energy of an $n$-layer slab, $E_{\text {atom }}$ is the energy of a single Be atom, $N_{\text {at }}$ is the number of atoms per layer, and $n_{1}>n_{2}$ (see Fig. 4). This expression should converge to the cohesive energy if $n_{1}$ and $n_{2}$ are large enough, such that the slab mimics a bulk system.

Although the cohesive energy is of course a bulk property, the above definition of $E_{\text {coh }}$ requires the accurate description of a 2D "impurity." It should be noted though, that cohesive energies could of course be computed from a bulk and a singleatom calculation in DMC without the need of embedding. We use the above definition though to allow for a proof of principle that embedded DMC calculations of $2 \mathrm{D}$ periodic systems are possible.
TABLE II. Cohesive energies computed with DFT and DMC with and without embedding. When embedding is used, the cluster layers are in the center of the slab and the number of layers of the cluster is stated as $\tilde{n}_{i}$. The experimental value is given for comparison. The error bars in the DMC calculations stem nearly exclusively from the fit to different $k$ points and not from statistical sampling. The statistical error bars from sampling are below $2 \mathrm{meV}$ for all calculations.

\begin{tabular}{lccccc}
\hline \hline & $n_{2}$ & $n_{1}$ & $\tilde{n}_{2}$ & $\tilde{n}_{1}$ & $E_{\text {coh }}(\mathrm{eV})$ \\
\hline DFT & 10 & 12 & & & 3.72 \\
DFT & 10 & 11 & & & 3.72 \\
DFT (emb) & 11 & 12 & 1 & 2 & 3.72 \\
DFT & 1 & 2 & & & 3.63 \\
DMC & 10 & 12 & & & $3.39 \pm 0.01$ \\
DMC (emb) & 11 & 12 & 1 & 2 & $3.39 \pm 0.02$ \\
DMC (emb, $\left.\lambda=1 \times 10^{-6}\right)$ & 11 & 12 & 1 & 2 & $3.40 \pm 0.02$ \\
DMC & 1 & 2 & & & $3.30 \pm 0.01$ \\
DFT(PW91) [63] & & & & & 3.74 \\
Expt. [64] & & & & & 3.32 \\
\hline \hline
\end{tabular}

The slabs are cut with a (0001) surface termination from the hcp bulk of Be using $10 \AA$ for vacuum between adjacent images. We use pseudopotentials by Trail and Needs to describe the Be atoms. The DFT calculations are based on the PBE functional. We use periodic boundary conditions in all three spatial directions, and $20 \times 20 k$ points in the $x-y$ plane (i.e., plane of the slab) for $k$-point averaging. We employ the experimental lattice parameters $a=2.2866 \AA$ and $c=3.5833 \AA$ from Ref. [61], as well as a measured surface interlayer dilatation of 5.8\% [62] for the top and bottom layers (except for slabs with only one or two layers, see below).

For the DMC calculations we use periodic boundary conditions in the $x-y$ plane only, and a $3 \times 3$ slab supercell to limit finite-size effects. Additionally, we sample over $k$ points by using 5-7 random $k$-point shifts (twists) in the $x-y$ plane (i.e., in the plane of the slab). ${ }^{1}$ We obtain an approximation to the $k$-point converged DMC result $\bar{E}_{\mathrm{DMC}}$ from a linear fit

$$
E_{\mathrm{DMC}}\left(\mathbf{k}_{i}\right)=\bar{E}_{\mathrm{DMC}}+b\left(E_{\mathrm{DFT}}\left(\mathbf{k}_{i}\right)-\bar{E}_{\mathrm{DFT}}\right)
$$

of the DFT data obtained at a $k$ point $\mathbf{k}_{i}$ to the DMC energies obtained at the same $k$ point via the fit parameters $\bar{E}_{\mathrm{DMC}}$ and $b$, where $\bar{E}_{\mathrm{DFT}}$ is the $k$-point converged DFT result. This is similar to the correction scheme previously used for example in Ref. [43].

For the computation of the energy of a single atom $E_{\text {atom }}$ needed for Eq. (15), we used a box of $14 \times 14 \times 14 \AA$ in DFT. In DMC, no periodicity was used for the atom in a box calculations. The DMC time step was 0.025 au in all cases.

First, we compute DFT and DMC reference values from slabs with $n_{1}=12$ and $n_{2}=10$. The resulting cohesive energies $E_{\text {coh }}$ are shown in Table II. The resulting DFT cohesive

\footnotetext{
${ }^{1}$ The number of twists used per geometry varies since some calculations were marked as outliers, either because the ratio $\frac{E_{\mathrm{VMC}}-E_{\mathrm{HF}}}{E_{\mathrm{DMC}}-E_{\mathrm{HF}}}$ was clearly worse than for other calculations, or because a certain twist clearly did not follow a linear relation between $E_{\mathrm{DFT}}\left(\mathbf{k}_{i}\right)$ and $E_{\mathrm{DMC}}\left(\mathbf{k}_{i}\right)$
} 


\section{$\mathrm{Xemb}(600 \mathrm{eV}) @ 600 \mathrm{eV}+\mathrm{emb}(600 \mathrm{eV}) @ 1720 \mathrm{eV}$ \\ +emb(1000eV)@1000eV * emb(1000eV)@1720eV}

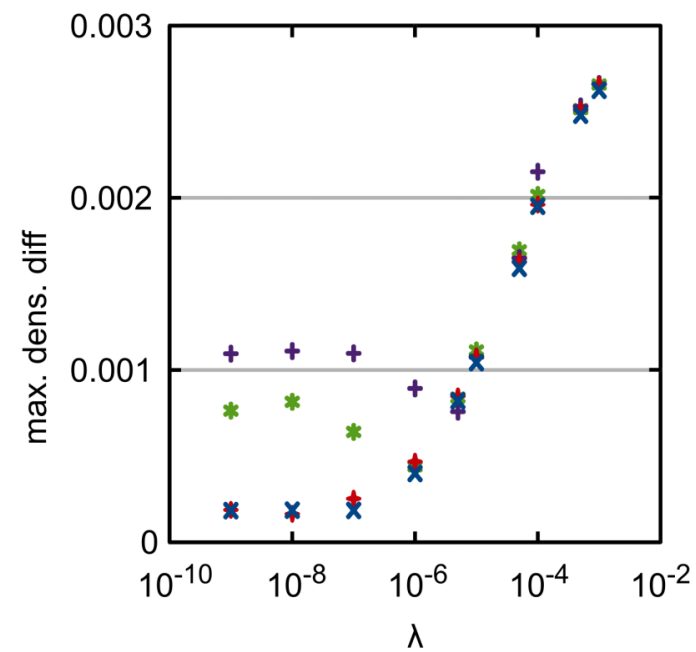

FIG. 5. Absolute values of the maximum density differences between the full and the embedded system obtained for a 6 layer Be slab at different values of $\lambda$.

energies $E_{\text {coh }}$ (see Table II) correspond well to previous DFT(PW91) results by Wachowitz et al. [63] ${ }^{2}$ while the DMC calculations agree much better with the experiment.

The next step is to find a converged embedding potential for Be slabs. Since we want to use the embedding in DMC, "converged" in this case also means transferable to higher $E_{\text {cut }}$. In order to investigate the convergence of the embedding potential, we first use two different toy systems. The first has three layers only, where the two outermost layers belong to the environment and the central layer constitutes the cluster. The second toy system has six layers, with the center two layers belonging to the cluster. The electronic partitioning is, in all cases, chosen such that the fragments remain neutral. For these toy systems, we find that, at fixed $E_{\text {cut }}$, untypically low values of $\lambda$ [see Eq. (9)] lead to a better convergence of the maximum density difference. Ported to higher $E_{\text {cut }}$, however, the same embedding potential typically gives much higher maximum and integral density differences (Fig. 5). Using higher values of $E_{\text {cut }}$ right away when designing the embedding potential seems to alleviate the problem to a certain extent, but this again becomes a question of computational cost.

One can understand the better convergence of the embedding potential with decreasing $\lambda$ by looking at the resulting embedding potential shown in Fig. 6 . The broad regions of negative embedding potential (indicated by arrows in Fig. 6) induce binding between the cluster and the environment layers. In addition to these broad structures, however, strong local fluctuations are apparent at the Be-atom sites close to the cluster - environment boundary: a positive peak in the embedding potential pushes density away from the nuclei to regions above

\footnotetext{
${ }^{2}$ Note that the PBE functional was designed to reproduce PW91 results, such that we may expect similar results for PW91 and PBE for the Be system too.
}

(a)

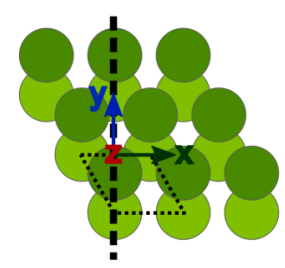

(b)
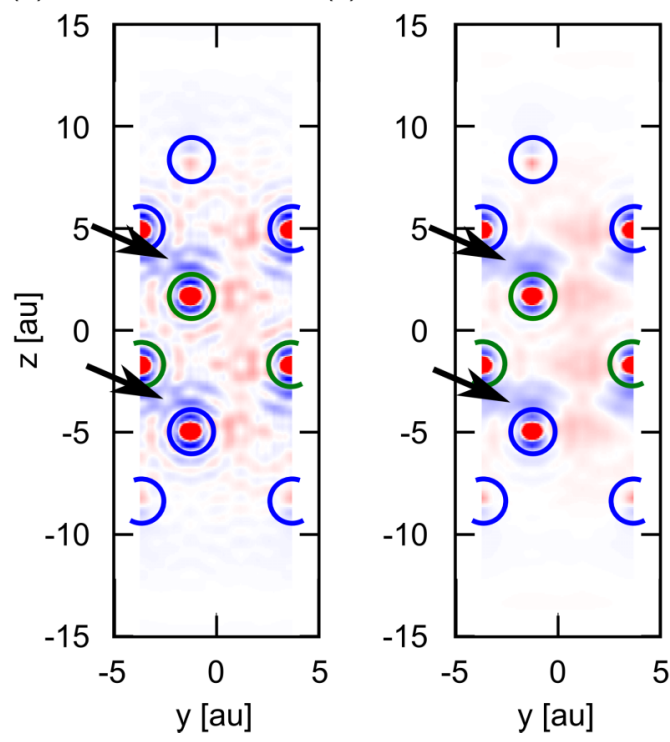

0.4

FIG. 6. 2D cut through the optimized embedding potential for a six-layer slab of beryllium, where the two outermost layers on the top and the two outermost layers on the bottom are described by the environment and the two central layers are cluster atoms. (a) Sketch of the geometry. The direction of the 2D cut used in (b) and (c) is indicated by a bold dashed line. Dark green balls indicate first-layer atoms, light green balls second-layer atoms. The thin dotted line shows the unit cell. (b) Embedding potential obtained using $\lambda=1 \times 10^{-6}$; black arrows indicate the region in the embedding potential expected to be responsible to replicate the binding between the cluster and the environment. (c) Same as (b) but using an $\mathbf{r}$-dependent $\lambda$ function with $\lambda_{i}=1 \times 10^{-7}, \lambda_{o}=1 \times 10^{-5}$, and $r_{\text {cut }}=1.0 \AA$. The blue and green circles represent the positions of the environment and cluster atoms in the cut plane, respectively.

and below the atoms, characterized by negative values in the embedding potential. It seems likely that these pronounced structures in the embedding potential are not due to numerical noise, but rather represent a change of orbital structure due to the binding (from $s$ like to more $p$ like).

A large value of $\lambda$ will not only suppress nonphysical oscillations in the potential, but will also penalize physical variations in the embedding potential, thus degrading the quality of the embedding at the nuclei. For constant $\lambda$ one is thus stuck between two choices: either one uses a large value of $\lambda$, which hampers convergence while suppressing nonphysical oscillations, or one chooses a low value of $\lambda$, at the price of introducing large small-scale oscillations. We believe that such a behavior is not unique to this system but will be found in all cases where the orbital structure of an atom is strongly influenced by the binding to the environment, such as also observed in most covalently bound systems. To allow for appropriately structured embedding potentials where the inter- 

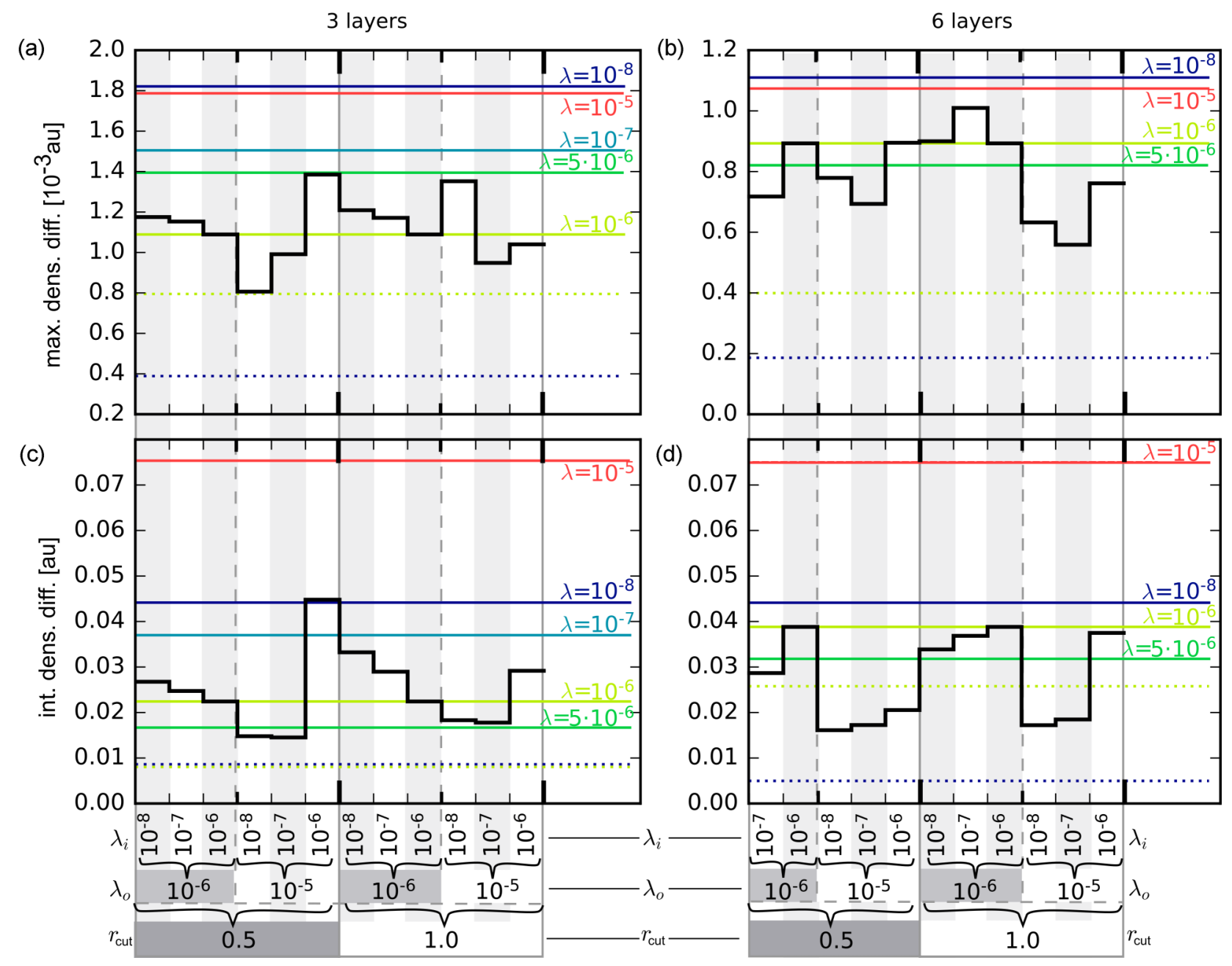

FIG. 7. Maximum and integrated density difference for a three- and a six-layer slab of beryllium for different values of $\lambda$. Since the density differences at high and at low plane-wave cutoff should both be low, we only report the larger of the differences obtained for $E_{\text {cut }}=600 \mathrm{eV}$ or for an embedding potential computed at $E_{\text {cut }}=600 \mathrm{eV}$ and used at $E_{\text {cut }}=1720 \mathrm{eV}$, unless noted otherwise. Horizontal lines: values obtained for constant values of $\lambda\left(\lambda_{o}=\lambda_{i}\right)$; dotted horizontal lines: result obtained at $E_{\text {cut }}=600 \mathrm{eV}$ (shown for reference at $\lambda=1 \times 10^{-8}$ and $\lambda=1 \times 10^{-6}$ only); thick black line: results obtained for different combinations of $\lambda_{i}, \lambda_{o}$, and $r_{\text {cut }}$ (Bohr).

action between subsystems dictates strong density variations, while still sufficiently penalizing nonphysical oscillations deep within the cluster, we modify the embedding code to allow for an $\mathbf{r}$ dependent $\lambda$ that varies smoothly from $\lambda_{i}$ in the area close to the atoms to $\lambda_{o}$ away from the atom:

$$
\begin{gathered}
\lambda(\mathbf{r})=\lambda_{i}^{f\left(\tilde{r}_{\mathrm{at}}^{2}\right)} \lambda_{o}^{1-f\left(\tilde{r}_{\mathrm{at}}^{2}\right),} \\
\tilde{r}_{\mathrm{at}}(\mathbf{r})=\frac{r_{\mathrm{na}}(\mathbf{r})}{r_{\mathrm{cut}}},
\end{gathered}
$$

where $r_{\text {na }}$ defines the distance from $\mathbf{r}$ to the nearest atom and the cutoff function $f$ is defined as

$$
f(x)= \begin{cases}e^{-\frac{x}{1-x}}, & x<1 \\ 0, & x \geqslant 1\end{cases}
$$

Using the three- and the six-layer systems, we performed extensive tests using different values of $r_{\text {cut }}, \lambda_{i}$, and $\lambda_{o}$, also accounting for the changes in the density difference when going to a higher value of $E_{\text {cut }}$. Judging from the maximum and the integrated density differences, the $\mathbf{r}$-dependent $\lambda$ approach can improve the convergence of the embedding potential (see Fig. 7): In general, the combination of relatively large $\lambda_{o}$ values of $1 \times 10^{-5}$ with $\lambda_{i}$ values a few orders of magnitudes smaller gives good results, as characterized by maximum and integrated density differences generally smaller than those obtained for the best result for constant $\lambda$. Furthermore, compared to a constant $\lambda=1 \times 10^{-6}$, which yields relatively good results in terms of maximum and integrated density differences at both low and high $E_{\text {cut }}$ (see Fig. 7), a spatially varying $\lambda$ additionally allows for an efficient suppression of oscillations in the intermediate region (see Fig. 6). Larger constant values of $\lambda \geqslant 1 \times 10^{-5}$ would also suppress these spurious oscillations but yield poor agreement with the reference density due to the uniformly suppressed oscillations. For the system at hand, we therefore conclude that only a spatially varying $\lambda$ results in the selective suppression of oscillations in the intermediate region while at the same time retaining the physical oscillations close to the core and thus allows for a stable and physical convergence of the embed- 
ding potential. We choose $r_{\text {cut }}=1.0$ a.u., $\lambda_{o}=1 \times 10^{-5}$, and $\lambda_{i}=1 \times 10^{-7}$, allowing for small maximum and integrated density differences, while also resulting in an embedding potential with strongly suppressed nonphysical oscillations far away from the atomic cores [Figs. 7 and 6(c)]. While probably not unique (compare Fig. 7), these parameters appeared appropriate for both systems under investigation (three-layer and six-layer slab). They were therefore subsequently used to create a DFT embedding potential replacing the outermost five layers on the top and on the bottom and leaving the central layers as active cluster (Fig. 4).

Using slabs with $n_{2}=11$ and $n_{1}=12$, and $\tilde{n}_{2}=1$ and $\tilde{n}_{1}=2$ active layers, respectively, we recalculated the cohesive energy for both DFT and DMC. The resulting DFT calculations fit perfectly to the previous calculations for $n_{2}=10$ and $n_{1}=12$ (see Table II) (note that the embedded DFT calculation per definition gives the same energy as the full system, so this result simply shows that the cohesive energy does not depend on whether $n_{1}=12$ or 11 ). By contrast, DFT calculations based on thin slabs with $n_{2}=1$ and $n_{1}=2$ underestimate the cohesive energy by $0.1 \mathrm{eV}$ compared to the converged DFT calculations with $n_{2}=11$ and $n_{1}=12$ or $n_{2}=10$ and $n_{1}=12$. This clearly indicates that the embedding potential is necessary in order to obtain correct results and that, if a match is observed in DMC, this is not purely coincidental. Indeed, the results of the embedded DMC calculations for $\tilde{n}_{2}=1$ and $\tilde{n}_{1}=2$ agree very well with the DMC calculation of the full system with $n_{2}=10$ or $n_{1}=12$. We can thus obtain correct results from a much cheaper computational setup with the number of atoms reduced by an order of magnitude.

As a cross-check of whether the embedding potential is actually necessary, we performed DMC calculations for $n_{2}=$ 1 and $n_{1}=2$. As for the DFT results, we observe a shift in cohesive energy by $0.1 \mathrm{eV}$, clearly showing the necessity of embedding (note that for a fair comparison, the reference has to be the converged DMC calculation with $n_{2}=10$ and $n_{1}=12$ and not the experimental results).

As a final check for the sensitivity of the embedded DMC results on the exact embedding potential, we also calculated the DMC energy for an embedding potential constructed at a constant damping factor of $\lambda=1 \times 10^{-6}$. The resulting DMC cohesive energy (see Table II) lies within error bars of the previously obtained DMC value, indicating that the DMC is not overly sensitive to "wiggles" in the embedding potential. As discussed above, this agreement may be rationalized based on the argument that fast oscillations in the wave function will be suppressed due to the associated high kinetic energy contributions. It should be noted, however, that such an agreement may well depend on the plane-wave cutoff used in the initial construction of the embedding potential since the spatial extent of the fluctuations in the embedding potential may depend on this parameter.

Overall, our results show that periodic embedding is possible in DMC and that it can give excellent and reliable results with much reduced computational cost.

\section{SUMMARY AND CONCLUSION}

We have implemented a potential functional embedding for quantum Monte Carlo in the CASINO code. The applicability of potential functional embedding to DMC was tested using two simple test systems: an $\mathrm{H}$ chain and a Be slab. We show that high-quality results are obtainable when using the embedding approach in DMC in spite of the "ripples" that are typically present in such embedding potentials. The embedded DMC results were especially good for the Be slab for which the embedded DMC calculations reproduced the experimental value and the DMC result for the cohesive energy very well. Embedded DMC thus presents an alternative to embedded wave-function methods in cases where the necessary cluster size is too large for conventional quantum chemistry or where basis-set superposition errors become too large to be efficiently corrected. This may be of particular interest when studying the interaction of molecules with metal surfaces, such as dissociative adsorption reactions.

Furthermore, we have shown how embedded DMC can be used for systems in which the cluster of interest is periodic. This approach is of great interest when studying, for example, quasi-2D superconducting materials, overlayer structures, and other phenomena that explicitly require periodicity in one or two dimensions. This possibility to treat periodic, embedded systems is new, since conventional wave-function-based quantum chemistry codes typically do not allow for periodicity (although exceptions exist $[5,33,65]$ ).

Along the way, we have also developed a slightly altered stabilization for the self-consistent development of embedding potentials. We believe that the proposed setup is suitable for systems in which the binding strongly influences the electronic structure and may thus provide a general improvement of the embedding optimization.

\section{ACKNOWLEDGMENTS}

We thank E. Carter and her group for providing us with their embedding codes used in this work. Furthermore, we thank P. López for checking our coding for the CASINO code and preparing it for inclusion into the CASINO program package. The authors acknowledge financial support from the European Research Council through an ERC-2013 Advanced Grant (No. 338580) and from FWF (Austria) Grant No. SFB-041 ViCoM.
[1] T. Janowski, A. R. Ford, and P. Pulay, J. Chem. Theory Comput. 3, 1368 (2007).

[2] M. Schütz and H.-J. Werner, J. Chem. Phys. 114, 661 (2000).

[3] W. A. Lester, Jr., L. Mitas, and B. Hammond, Chem. Phys. Lett. 478, 1 (2009).

[4] W. M. C. Foulkes, L. Mitas, R. J. Needs, and G. Rajagopal, Rev. Mod. Phys. 73, 33 (2001).
[5] G. H. Booth, A. Grüneis, G. Kresse, and A. Alavi, Nature (London) 493, 365 (2013).

[6] J. P. Perdew, A. Ruzsinszky, G. I. Csonka, L. A. Constantin, and J. Sun, Phys. Rev. Lett. 103, 026403 (2009).

[7] P. Haas, F. Tran, P. Blaha, and K. Schwarz, Phys. Rev. B 83, 205117 (2011). 
[8] J. Sun, R. Haunschild, B. Xiao, I. W. Bulik, G. E. Scuseria, and J. P. Perdew, J. Chem. Phys. 138, 044113 (2013).

[9] J. Sun, A. Ruzsinszky, and J. P. Perdew, Phys. Rev. Lett. 115, 036402 (2015).

[10] J. Tao and Y. Mo, Phys. Rev. Lett. 117, 073001 (2016).

[11] H. S. Yu, X. He, and D. G. Truhlar, J. Chem. Theory Comput. 12, 1280 (2016).

[12] M. Dion, H. Rydberg, E. Schröder, D. C. Langreth, and B. I. Lundqvist, Phys. Rev. Lett. 92, 246401 (2004).

[13] J. Wellendorff, K. T. Lundgaard, A. Møgelhøj, V. Petzold, D. D. Landis, J. K. Nørskov, T. Bligaard, and K. W. Jacobsen, Phys. Rev. B 85, 235149 (2012).

[14] A. Tkatchenko, R. A. DiStasio, R. Car, and M. Scheffler, Phys. Rev. Lett. 108, 236402 (2012).

[15] K. T. Lundgaard, J. Wellendorff, J. Voss, K. W. Jacobsen, and T. Bligaard, Phys. Rev. B 93, 235162 (2016).

[16] J. Paier, B. G. Janesko, T. M. Henderson, G. E. Scuseria, A. Grüneis, and G. Kresse, J. Chem. Phys. 132, 094103 (2010).

[17] B. G. Janesko, G. Scalmani, and M. J. Frisch, J. Chem. Phys. 141, 034103 (2014).

[18] J. C. Snyder, M. Rupp, K. Hansen, K.-R. Müller, and K. Burke, Phys. Rev. Lett. 108, 253002 (2012).

[19] S. E. Derenzo, M. K. Klintenberg, and M. J. Weber, J. Chem. Phys. 112, 2074 (2000).

[20] E. R. Batista and R. A. Friesner, J. Phys. Chem. B 106, 8136 (2002).

[21] A. Warshel and M. Levitt, J. Mol. Biol. 103, 227 (1976).

[22] T. A. Wesolowski and A. Warshel, J. Phys. Chem. 97, 8050 (1993).

[23] S. Fux, C. R. Jacob, J. Neugebauer, L. Visscher, and M. Reiher, J. Chem. Phys. 132, 164101 (2010).

[24] C. Huang, M. Pavone, and E. A. Carter, J. Chem. Phys. 134, 154110 (2011).

[25] C. Huang and E. A. Carter, J. Chem. Phys. 135, 194104 (2011).

[26] C. Daday, C. König, J. Neugebauer, and C. Filippi, Chem. Phys. Chem. 15, 3205 (2014).

[27] G. Knizia and G. K.-L. Chan, J. Chem. Theory Comput. 9, 1428 (2013).

[28] I. W. Bulik, W. Chen, and G. E. Scuseria, J. Chem. Phys. 141, 054113 (2014).

[29] I. W. Bulik, G. E. Scuseria, and J. Dukelsky, Phys. Rev. B 89, 035140 (2014).

[30] F. R. Manby, M. Stella, J. D. Goodpaster, and T. F. Miller, J. Chem. Theory Comput. 8, 2564 (2012).

[31] J. D. Goodpaster, T. A. Barnes, F. R. Manby, and T. F. Miller III, J. Chem. Phys. 140, 18A507 (2014).

[32] F. Libisch, M. Marsman, J. Burgdörfer, and G. Kresse, J. Chem. Phys. 147, 034110 (2017).

[33] D. V. Chulhai and J. D. Goodpaster, J. Chem. Theory Comput. 14, 1928 (2018).

[34] R. Guareschi, H. Zulfikri, C. Daday, F. M. Floris, C. Amovilli, B. Mennucci, and C. Filippi, J. Chem. Theory Comput. 12, 1674 (2016).

[35] K. A. Schwarz, R. Sundararaman, K. Letchworth-Weaver, T. A. Arias, and R. G. Hennig, Phys. Rev. B 85, 201102 (2012).
[36] F. Libisch, C. Huang, and E. A. Carter, Acc. Chem. Res. 47, 2768 (2014).

[37] F. Libisch, C. Huang, P. Liao, M. Pavone, and E. A. Carter, Phys. Rev. Lett. 109, 198303 (2012).

[38] S. Mukherjee, F. Libisch, N. Large, O. Neumann, L. V. Brown, J. Cheng, J. B. Lassiter, E. A. Carter, P. Nordlander, and N. J. Halas, Nano Lett. 13, 240 (2013).

[39] N. Nemec, M. D. Towler, and R. J. Needs, J. Chem. Phys. 132, 034111 (2010).

[40] F. R. Petruzielo, J. Toulouse, and C. J. Umrigar, J. Chem. Phys. 136, 124116 (2012).

[41] G.-J. Kroes, J. Phys. Chem. Lett. 6, 4106 (2015).

[42] S. Mallikarjun Sharada, T. Bligaard, A. C. Luntz, G.-J. Kroes, and J. K. Nørskov, J. Phys. Chem. C 121, 19807 (2017).

[43] K. Doblhoff-Dier, J. Meyer, P. E. Hoggan, and G.-J. Kroes, J. Chem. Theory Comput. 13, 3208 (2017).

[44] A. J. R. Hensley, K. Ghale, C. Rieg, T. Dang, E. Anderst, F. Studt, C. T. Campbell, J.-S. McEwen, and Y. Xu, J. Phys. Chem. C 121, 4937 (2017).

[45] C. R. Jacob, J. Chem. Phys. 135, 244102 (2011).

[46] R. J. Needs, M. D. Towler, N. D. Drummond, and P. L. Ríos, J. Phys.: Condens. Matter 22, 023201 (2010).

[47] J. Kolorenč and L. Mitas, Rep. Prog. Phys. 74, 026502 (2011).

[48] C. Filippi and S. Fahy, J. Chem. Phys. 112, 3523 (2000).

[49] E. Giner, A. Scemama, and M. Caffarel, J. Chem. Phys. 142, 044115 (2015).

[50] M. C. Per and D. M. Cleland, J. Chem. Phys. 146, 164101 (2017).

[51] C. J. Umrigar and C. Filippi, Phys. Rev. Lett. 94, 150201 (2005).

[52] N. D. Drummond and R. J. Needs, Phys. Rev. B 72, 085124 (2005).

[53] J. Toulouse and C. J. Umrigar, J. Chem. Phys. 126, 084102 (2007).

[54] C. J. Umrigar, J. Toulouse, C. Filippi, S. Sorella, and R. G. Hennig, Phys. Rev. Lett. 98, 110201 (2007).

[55] Q. Wu and W. Yang, J. Chem. Phys. 118, 2498 (2003).

[56] J. Cheng, Embedding Theory: Applications and Development, $\mathrm{Ph} . \mathrm{D}$. thesis, Princeton University, 2016.

[57] C. Daday, C. König, O. Valsson, J. Neugebauer, and C. Filippi, J. Chem. Theory Comput. 9, 2355 (2013).

[58] L. K. Wagner, Phys. Rev. B 92, 161116 (2015).

[59] J. P. Perdew and A. Zunger, Phys. Rev. B 23, 5048 (1981).

[60] J. P. Perdew, K. Burke, and M. Ernzerhof, Phys. Rev. Lett. 77, 3865 (1996).

[61] D. R. Schwarzenberger, Philos. Mag. 4, 1242 (1959).

[62] H. L. Davis, J. B. Hannon, K. B. Ray, and E. W. Plummer, Phys. Rev. Lett. 68, 2632 (1992).

[63] E. Wachowicz and A. Kiejna, J. Phys.: Condens. Matter 13, 10767 (2001).

[64] C. Kittel, Introduction to Solid State Physics, 8th ed. (John Wiley \& Sons, New York, 2005), p. 50.

[65] R. Dovesi, A. Erba, R. Orlando, C. M. Zicovich-Wilson, B. Civalleri, L. Maschio, M. Réat, S. Casassa, J. Baima, S. Salustro, and B. Kirtman, WIREs Comput. Mol. Sci. 8, e1360 (2018). 